\title{
THE METHOD OF THE HYPERCIRCLE IN ELASTICITY WHEN BODY FORCES ARE PRESENT*
}

\author{
By
}

J. L. SYNGE

Carnegie Institute of Technology

In an earlier paper ${ }^{1}$ the method of the hypercircle in function space for boundary value problems in elasticity was developed under the restriction that body forces are absent. The purpose of the present note is to remove that restriction, and present the method in complete form, using the notation and basic ideas of PS.

Let an elastic body (in general anisotropic) be subjected to body forces $X_{i}$ per unit volume, so that the equations of equilibrium are

$$
E_{i, j}+X_{i}=0 \text {. }
$$

As regards boundary conditions, we shall suppose that the surface $B$ of the body may be divided into regions designated by

$$
\left[u_{n}, u_{t}\right], . \quad\left[u_{n}, T_{t}\right], \quad\left[u_{t}, T_{n}\right], \quad\left[T_{n}, T_{t}\right] .
$$

Here $\left[u_{n}, u_{t}\right]$ indicates a portion of $B$ on which both the normal component $u_{n}$ of displacement and the tangential (vector) component $u_{t}$ of displacement are assigned; $\left[u_{n}, T_{t}\right]$ indicates a portion of $B$ on which the normal component of displacement and the tangential component of stress are assigned; and so on.

As in PS, a state of stress throughout the body corresponds to a vector in function space, and the scalar product is defined by $\mathbf{S}^{\prime} \cdot \mathbf{S}^{\prime \prime}=\int e_{i j}^{\prime} E_{i j}^{\prime \prime} d v$, the integral being taken throughout the body. We set up the following notation:

$\mathbf{S}=$ natural state satisfying the equations of equilibrium and compatibility, and also the boundary conditions.

$\mathbf{S}^{\prime}=$ basic stress state, satisfying the equations of equilibrium

$$
E_{i j, j}^{\prime}+X_{i}=0,
$$

and all boundary conditions on stress; this means that

$$
\begin{aligned}
& T_{t}^{\prime}=T_{t} \text { on }\left[u_{n}, T_{t}\right], \\
& T_{n}^{\prime}=T_{n} \text { on }\left[u_{t}, T_{n}\right], \\
& T_{n}^{\prime}=T_{n} \text { and } T_{t}^{\prime}=T_{t} \text { on }\left[T_{n}, T_{t}\right] .
\end{aligned}
$$

$\mathbf{I}_{p}^{\prime}(p=1,2, \cdots m)=$ orthonormal homogeneous stress states, each satisfying the equations of equilibrium without body forces,

$$
E_{(p) i j, j}^{\prime}=0,
$$

and making $T_{(p) n}^{\prime}$ vanish wherever $T_{n}$ is assigned and making $T_{(p) t}^{\prime}$ vanish wherever $T_{t}$ is assigned.

$\mathbf{S}^{\prime \prime}=$ basic displacement state; this corresponds to a state of stress which satisfies

* Received Aug. 25, 1947.

1 W. Prager and J. L. Synge, Quarterly of Applied Mathematics, 5, 241-271 (1947); this paper will be referred to as PS. $C f$. also J. L. Synge, The method of the hypercircle in function space for boundary value problems, Proc. Roy. Soc. London (A) 191, 447-467 (1947). 
the equations of compatibility, so that a displacement $u_{j}^{\prime \prime}$ exists. This displacement satisfies all boundary conditions on displacement, so that

$$
\begin{aligned}
& u_{n}^{\prime \prime}=u_{n} \text { and } u_{t}^{\prime \prime}=u_{t} \text { on }\left[u_{n}, u_{t}\right], \\
& u_{n}^{\prime \prime}=u_{n} \text { on }\left[u_{n}, T_{t}\right], \\
& u_{t}^{\prime \prime}=u_{t} \text { on }\left[u_{t}, T_{n}\right] .
\end{aligned}
$$

$\mathbf{I}_{q}^{\prime \prime}(q=1,2, \cdots n)=$ orthonormal homogeneous displacement states, each corresponding to a state of stress satisfying the equations of compatibility, so that a displacement $u_{(q)}^{\prime \prime}$ exists; this displacement is such that $u_{(q) n}^{\prime \prime}$ vanishes wherever $u_{n}$ is assigned and $u_{(\mathbf{q}) t}^{\prime \prime}$ vanishes wherever $u_{t}$ is assigned.

It is important to note that, if $k^{\prime}$ and $k^{\prime \prime}$ are any constants, then $\mathbf{S}^{\prime}+k^{\prime} \mathbf{I}_{p}^{\prime}$ belongs to the class of basic stress states $\left(\mathbf{S}^{\prime}\right)$ and $\mathbf{S}^{\prime \prime}+k^{\prime \prime} \mathbf{I}_{q}^{\prime \prime}$ belongs to the class of basic displacement states $\left(\mathbf{S}^{\prime \prime}\right)$.

We now proceed to prove that the extremity of $\mathbf{S}$ lies on a hypersphere determined by $\mathbf{S}^{\prime}$ and $\mathbf{S}^{\prime \prime}$. By Green's theorem we have

$$
\begin{aligned}
\mathbf{S}^{\prime} \cdot \mathbf{S}^{\prime \prime} & =\int e_{i j}^{\prime \prime} E_{i j}^{\prime} d v=\int u_{i, j}^{\prime \prime} E_{i j}^{\prime} d v \\
& =\int u_{i}^{\prime \prime} T_{i}^{\prime} d B-\int u_{i}^{\prime \prime} E_{i j, j}^{\prime} d v,
\end{aligned}
$$

wnere $d B$ is an element of the bounding surface $B$. Hence, by (3),

$$
\mathbf{S}^{\prime} \cdot \mathbf{S}^{\prime \prime}=\int u_{i}^{\prime \prime} T_{i}^{\prime} d B+\int u_{i}^{\prime \prime} X_{i} d v .
$$

But $\mathbf{S}$ belongs to the class of $\mathbf{S}^{\prime}$ and also to the class of $\mathbf{S}^{\prime \prime}$. Thus we can substitute $\mathbf{S}$ for $\mathbf{S}^{\prime}$ or $\mathbf{S}^{\prime \prime}$ in (7) and so obtain the following equations:

$$
\begin{aligned}
\mathbf{S} \cdot \mathbf{S}^{\prime \prime} & =\int u_{i}^{\prime \prime} T_{i} d B+\int u_{i}^{\prime \prime} X_{i} d v, \\
\mathbf{S} \cdot \mathbf{S} & =\int u_{i} T_{i}^{\prime} d B+\int u_{i} X_{i} d v, \\
\mathbf{S} \cdot \mathbf{S} & =\int u_{i} T_{i} d B+\int u_{i} X_{i} d v .
\end{aligned}
$$

By addition and subtraction of (7) and (8), we obtain

$$
\mathbf{S} \cdot \mathbf{S}-\mathbf{S} \cdot \mathbf{S}^{\prime}-\mathbf{S} \cdot \mathbf{S}^{\prime \prime}+\mathbf{S}^{\prime} \cdot \mathbf{S}^{\prime \prime}=\int\left(u_{i}-u_{i}^{\prime \prime}\right)\left(T_{i}-T_{i}^{\prime}\right) d B
$$

For any pair of vectors, $U_{j}, V_{j}$, we have

$$
\int U_{i} V_{i} d B=\int U_{n} V_{n} d B+\int U_{t} V_{t} d B
$$

where $U_{n}, V_{n}$ are normal components, and $U_{t} V_{t}$ is the ordinary scalar product of the tangential vector components. We apply this reduction to the right hand side of (9), 
and then split each integral into four parts corresponding to the four regions (2). It is easily seen from (4) and (6) that the result is zero, and so (9) reads

$$
\mathbf{S} \cdot \mathbf{S}-\mathbf{S} \cdot \mathbf{S}^{\prime}-\mathbf{S} \cdot \mathbf{S}^{\prime \prime}+\mathbf{S}^{\prime} \cdot \mathbf{S}^{\prime \prime}=0,
$$

or, equivalently,

$$
\left[\mathbf{S}-\frac{1}{2}\left(\mathbf{S}^{\prime}+\mathbf{S}^{\prime \prime}\right)\right]^{2}=\frac{1}{4}\left(\mathbf{S}^{\prime}-\mathbf{S}^{\prime \prime}\right)^{2} .
$$

This formula locates the extremity of $\mathbf{S}$ on a hypersphere in function space with center at

$$
\mathbf{C}_{0}=\frac{1}{2}\left(\mathbf{S}^{\prime}+\mathbf{S}^{\prime \prime}\right)
$$

and with radius $R$ given by

$$
R^{2}=\frac{1}{4}\left(\mathbf{S}^{\prime}-\mathbf{S}^{\prime \prime}\right)^{2} .
$$

Since all that is required of $\mathbf{S}^{\prime}$ and $\mathbf{S}^{\prime \prime}$ is that they belong respectively to the classes of basic stress states and basic displacement states, it follows from a remark made earlier that we may make the following substitutions in (11):

$$
\begin{aligned}
& \text { for } \mathbf{S}^{\prime} \text { substitute } \mathbf{S}^{\prime}+k^{\prime} \mathbf{I}_{p}^{\prime}, \\
& \text { for } \mathbf{S}^{\prime \prime} \text { substitute } \mathbf{S}^{\prime \prime}+k^{\prime \prime} \mathbf{I}_{q}^{\prime \prime},
\end{aligned}
$$

where $k^{\prime}, k^{\prime \prime}$ are arbitrary constants. When we do this, and then subtract (11) from the result, we get an equation which must be satisfied for all values of $k^{\prime}, k^{\prime \prime}$. This yields

$$
\left.\begin{array}{rl}
\mathbf{S} \cdot \mathbf{I}_{p}^{\prime}=\mathbf{S}^{\prime \prime} \cdot \mathbf{I}_{p}^{\prime} & (p=1,2, \cdots, m), \\
\mathbf{S} \cdot \mathbf{I}_{q}^{\prime \prime}=\mathbf{S}^{\prime} \cdot \mathbf{I}_{q}^{\prime \prime} & (q=1,2, \cdots, n),
\end{array}\right\}
$$

and also

$$
\mathbf{I}_{p}^{\prime} \cdot \mathbf{I}_{q}^{\prime \prime}=0 \quad(p=1,2, \cdots, m ; q=1,2, \cdots, n) ;
$$

this last result shows that the set of homogeneous stress vectors is orthogonal to the set of homogeneous displacement vectors.

Equations (11) and (15) locate the extremity of the natural vector $\mathbf{S}$ on a hypercircle $\Gamma$. It is easy to prove that the center $\mathbf{C}$ of $\Gamma$ and its radius $R$ are given by

$$
\begin{aligned}
\mathbf{C} & =\frac{1}{2}\left[\mathbf{S}^{\prime}+\mathbf{S}^{\prime \prime}-\sum_{p=1}^{m} \mathbf{I}_{\rho}^{\prime}\left\{\left(\mathbf{S}^{\prime}-\mathbf{S}^{\prime \prime}\right) \cdot \mathbf{I}_{p}^{\prime}\right\}+\sum_{q=1}^{n} \mathbf{I}_{q}^{\prime \prime}\left\{\left(\mathbf{S}^{\prime}-\mathbf{S}^{\prime \prime}\right) \cdot \mathbf{I}_{q}^{\prime \prime}\right\}\right], \\
R^{2} & =\frac{1}{4}\left[\left(\mathbf{S}^{\prime}-\mathbf{S}^{\prime \prime}\right)^{2}-\sum_{p=1}^{m}\left\{\left(\mathbf{S}^{\prime}-\mathbf{S}^{\prime \prime}\right) \cdot \mathbf{I}_{p}^{\prime}\right\}^{2}-\sum_{q=1}^{n}\left\{\left(\mathbf{S}^{\prime}-\mathbf{S}^{\prime \prime}\right) \cdot \mathbf{I}_{q}^{\prime \prime}\right\}^{2}\right] .
\end{aligned}
$$

Let us use $\mathrm{I}_{r}(r=1,2, \cdots, m+n)$ to denote the whole set of homogeneous vectors $\mathbf{I}_{p}^{\prime} ; \mathbf{I}_{q}^{\prime \prime \prime}$, so that, by (16), $\mathbf{I}_{r}$ form a set of orthonormal vectors in function space. The equation of the hypercircle $\Gamma$ may be written

$$
\mathbf{S}=\mathbf{C}+R \mathbf{J},
$$

where $\mathrm{J}$ is arbitrary except for the equations

$$
\mathrm{J}^{2}=1, \quad \mathrm{~J} \cdot \mathrm{I}_{r}=0 \quad(r=1,2, \cdots, m+n) .
$$


We now seek the points on $\Gamma$ at minimum and maximum distances from the origin $O$ of function space. For any point $\mathbf{S}$ on $\Gamma$ we have, by (19),

$$
\mathbf{S}^{2}=\mathbf{C}^{2}+R^{2}+2 R \mathbf{C} \cdot \mathbf{J}
$$

and so, on account of (20), for stationary values of $\mathbf{S}^{2}$ we have

$$
\mathbf{C}=a \mathbf{J}+\sum_{r=1}^{m+n} b_{r} \mathbf{I}_{r}
$$

where $a$ and $b_{r}$ are undetermined multipliers. We deduce

$$
b_{r}=\mathbf{C} \cdot \mathbf{I}_{r},
$$

and

$$
a^{2}=\left[\mathbf{C}-\sum_{r=1}^{m+n} \mathbf{I}_{r}\left(\mathbf{C} \cdot \mathrm{I}_{r}\right)\right]^{2}=\mathbf{C}^{2}-\sum_{r=1}^{m+n}\left(\mathbf{C} \cdot \mathrm{I}_{r}\right)^{2}
$$

For a stationary value of $\mathbf{S}^{2}$ we must take, by (22) and (23),

$$
\mathbf{J}=a^{-1}\left[\mathbf{C}-\sum_{r=1}^{m+n} \mathbf{I}_{r}\left(\mathbf{C} \cdot \mathbf{I}_{r}\right)\right]
$$

We find it convenient to define a vector $\mathbf{G}$ by

$$
\mathbf{G}=\frac{1}{2}\left[\mathbf{S}^{\prime}+\mathbf{S}^{\prime \prime}-\sum_{r=1}^{m+n} \mathbf{I}_{r}\left\{\left(\mathbf{S}^{\prime}+\mathbf{S}^{\prime \prime}\right) \cdot \mathbf{I}_{r}\right\}\right],
$$

so that

$$
\mathbf{G}^{2}=\frac{1}{4}\left[\left(\mathbf{S}^{\prime}+\mathbf{S}^{\prime \prime}\right)^{2}-\sum_{r=1}^{m+n}\left\{\left(\mathbf{S}^{\prime}+\mathbf{S}^{\prime \prime}\right) \cdot \mathbf{I}_{r}\right\}^{2}\right] .
$$

By (17) and (26), we have

$$
\mathbf{C}=\mathbf{G}+\sum_{p=1}^{m} \mathbf{I}_{p}^{\prime}\left(\mathbf{S}^{\prime \prime} \cdot \mathbf{I}_{p}^{\prime}\right)+\sum_{q=1}^{n} \mathbf{I}_{q}^{\prime \prime}\left(\mathbf{S}^{\prime} \cdot \mathbf{I}_{q}^{\prime \prime}\right) .
$$

We note that $\mathbf{G} \cdot \mathbf{I}_{r}=0$, and so by (28)

$$
\mathbf{C} \cdot \mathbf{G}=\mathbf{G}^{2} \geqq 0 .
$$

By direct algebra we may prove that

$$
\mathbf{C}-\sum_{r=1}^{m+n} \mathbf{I}_{r}\left(\mathbf{C} \cdot \mathbf{I}_{r}\right)=\mathbf{G} .
$$

Thus, by (24), $a= \pm|\mathbf{G}|$, and, by (25), $\mathbf{J}= \pm \mathbf{G} /|\mathbf{G}|$. By (19) the stationary values of $\mathbf{S}^{2}$, if $\mathbf{S}$ ranges over $\Gamma$, correspond to the vectors

$$
\mathbf{C} \pm R \mathbf{G} /|\mathbf{G}| \text {. }
$$

Of these two vectors, that with the + sign is the greater, on account of (29). Accordingly, we may say that the point of $\Gamma$ farthest from the origin of function space has the position vector 


$$
\mathrm{V}^{\prime}=\mathbf{C}+R \mathbf{G} /|\mathbf{G}|
$$

and the point nearest to the origin has the position vector

$$
\mathbf{V}^{\prime \prime}=\mathbf{C}-R \mathbf{G} /|\mathbf{G}| .
$$

Thus we have the following inequalities for the magnitude of the vector $\mathbf{S}$ corresponding to the natural state of the elastic body:

$$
\mathrm{V}^{\prime \prime 2} \leqq \mathrm{~S}^{2} \leqq \mathrm{~V}^{\prime 2}
$$

or, by (29),

$$
\mathbf{C}^{2}+R^{2}-2 R|\mathbf{G}| \leqq \mathbf{S}^{2} \leqq \mathbf{C}^{2}+R^{2}+2 R|\mathbf{G}|,
$$

where $\mathbf{C}$ is given by (17), $R$ by (18), and $|\mathrm{G}|$ by (27), on taking the square root. We can show by direct algebra that

$$
\begin{aligned}
\mathbf{C}^{2}+R^{2}=\frac{1}{2}\left(\mathbf{S}^{\prime 2}+\mathbf{S}^{\prime \prime 2}\right)+\frac{1}{2} & {\left[\sum_{p=1}^{m}\left\{\left(\mathbf{S}^{\prime \prime} \cdot \mathbf{I}_{p}^{\prime}\right)^{2}-\left(\mathbf{S}^{\prime} \cdot \mathbf{I}_{p}^{\prime}\right)^{2}\right\}\right.} \\
& \left.+\sum_{q=1}^{n}\left\{\left(\mathbf{S}^{\prime} \cdot \mathbf{I}_{q}^{\prime \prime}\right)^{2}-\left(\mathbf{S}^{\prime \prime} \cdot \mathbf{I}_{q}^{\prime \prime}\right)^{2}\right\}\right] .
\end{aligned}
$$

The inequalities (34) or (35) bound the strain energy of the natural state below and above. It is interesting to note that the usual minimal principles bound the potential energy of the natural state, rather than its strain energy. 